\title{
Características dos casos de violência contra crianças e adolescentes do sexo feminino entre 10 e 19 anos notificados no Nordeste do Brasil (2015-2019)
}

Characteristics of cases of violence against female children and adolescents between 10 and 19

years reported in Northeastern Brazil (2015-2019)

Características de los casos de violencia niñas y adolescentes de entre 10 y 19 años denunciados en

el Noreste de Brasil (2015-2019)

Recebido: 07/06/2021 | Revisado: 14/06/2021 | Aceito: 19/06/2021 | Publicado: 25/06/2021

Paola Fernanda Santos Antunes ORCID: https://orcid.org/0000-0003-3031-1179

Universidade Tiradentes, Brasil

E-mail: paollaantuness24@gmail.com

Caique Anizio Santos da Rosa

ORCID: https://orcid.org/0000-0002-7507-2629

Universidade Tiradentes, Brasil

E-mail: caiquenizio@gmail.com

Izabella Mariane Ramos dos Santos

ORCID: https://orcid.org/0000-0002-3165-3798 Universidade Tiradentes, Brasil

E-mail: izabellamariane123@gmail.com

Mariana Silveira Silva

ORCID: https://orcid.org/0000-0002-4300-5739 Universidade Tiradentes, Brasil

E-mail: marianasilveirasilva15@gmail.com

Míria Dantas Pereira

ORCID: https://orcid.org/0000-0002-9774-9717 Universidade Tiradentes, Brasil

E-mail: miriadantaspereira@gmail.com

Mara Dantas Pereira

ORCID: https://orcid.org/0000-0002-5943-540X Universidade Federal de Sergipe, Brasil E-mail: maradantaspereira@gmail.com

\section{Resumo}

A violência contra a mulher trata-se de um relevante problema social e de saúde pública no mundo, tida como uma violação dos direitos humanos gerando danos à saúde física e mental da mulher. O presente artigo tem como objetivo descrever as características dos casos de violência contra crianças e adolescentes do sexo feminino entre 10 e 19 anos notificados na região Nordeste do Brasil nos anos de 2015 a 2019. Trata-se de um estudo ecológico, descritivo, e de caráter quantitativo desenvolvido por meio da análise de dados disponíveis no SINAN, acerca da população residente no Nordeste do Brasil, nos anos de 2015 a 2019. Utilizou-se o programa Microsoft Excel ${ }^{\circledR}$ versão 2019, para a organização e tabulação dos dados. Foram registradas 34.355 notificações de violência nos anos de 2015 a 2019. Houve prevalência das notificações das mulheres com idade de 15 a 19 anos, com 58,8\% (n= 20.185) e da cor parda $65,4 \%(n=22.463)$. Referente à escolaridade processou-se um maior número de notificações das mulheres com $5^{\mathrm{a}}$ a $8^{\mathrm{a}}$ série incompleta do Fundamental e Ensino médio incompleto, com 28,0\% ( $\mathrm{n}=9.565)$. Mediante dos achados, apontase para a importância da aplicabilidade de metodologias de segurança e prevenção das violências sofridas pelas mulheres, e ainda, para a construção de políticas públicas efetivas ao combate de violências contra crianças e adolescentes do sexo feminino, visto que elas se encontram em estado de vulnerabilidade.

Palavras-chave: Epidemiologia; Violência contra a mulher; Violência de gênero.

\begin{abstract}
Violence against women is a major social and public health problem worldwide, considered a violation of human rights and causing damage to women's physical and mental health. This article aims to describe the characteristics of cases of violence against female children and adolescents between 10 and 19 years old notified in the Northeast region of Brazil in the years 2015 to 2019. This is an ecological, descriptive, and quantitative study developed through the analysis of data available in SINAN, about the population residing in Northeast Brazil, in the years 2015 to 2019 . The Microsoft Excel ${ }^{\circledR}$ version 2019 program was used for the organization and tabulation of data. 34,355 notifications of violence were registered in the years 2015 to 2019. There was a prevalence of notifications of women aged 15 to 19
\end{abstract}


years old, with $58.8 \%(n=20,185)$ and of the brown color $65.4 \%(n=22,463)$. Regarding education, there was a higher number of notifications from women with 5th to 8th grade incomplete Elementary School and incomplete High School, with $28.0 \%(n=9,565)$. Given the findings, it points to the importance of the applicability of safety methodologies and prevention of violence suffered by women, and also, for the construction of effective public policies to combat violence against female children and adolescents, since they are in a state of vulnerability.

Keywords: Epidemiology; Violence against women; Gender-based violence.

\section{Resumen}

La violencia contra las mujeres es un importante problema social y de salud pública en todo el mundo, considerado como una violación de los derechos humanos y que causa daños a la salud física y mental de las mujeres. Este artículo tiene como objetivo describir las características de los casos de violencia contra niñas y adolescentes entre 10 y 19 años notificados en la región Nordeste de Brasil en los años 2015 a 2019. Este es un estudio ecológico, descriptivo y cuantitativo desarrollado a través del análisis de los datos disponibles en el SINAN, sobre la población residente en el Nordeste de Brasil, en los años 2015 a 2019. Para la organización y tabulación de los datos se utilizó el programa Microsoft Excel ${ }^{\circledR}$ versión 2019. En los años 2015 a 2019 se registraron 34.355 notificaciones de violencia. Hubo una prevalencia de las notificaciones de las mujeres con edad de 15 a 19 años, con un $58,8 \%(n=20.185)$ y de las mujeres con un $65,4 \%(n=22.463)$. En cuanto a la educación, hubo un mayor número de notificaciones de mujeres con $5^{\circ}$ a $8^{\circ}$ grado de Primaria incompleta y de Secundaria incompleta, con un 28,0\% ( $\mathrm{n}=9.565)$. Los hallazgos señalan la importancia de la aplicabilidad de metodologías de seguridad y prevención de la violencia sufrida por las mujeres, así como la construcción de políticas públicas efectivas para combatir la violencia contra las niñas y adolescentes, ya que se encuentran en estado de vulnerabilidad.

Palabras clave: Epidemiología; Violencia contra las Mujeres; Violencia de género.

\section{Introdução}

A Organização Mundial da Saúde (2002), caracteriza o termo violência como o deferimento intencional da força física e ameaças que acarretam consequências graves para si ou com o próximo, podendo agravar-se desde danos psicológicos ao óbito. Continuamente, em acréscimo à afirmativa supracitada, Minayo (2007), discorre que a violência consiste no reflexo das relações socioculturais estabelecidas em determinada ambiência, além dos aspectos comportamentais de cada indivíduo. Diante disso, compreende-se que a violência é uma problemática social e de saúde pública, além de caracterizar-se como um fator contribuinte e determinante para o desenvolvimento de doenças e agravos, transformando a saúde individual e coletiva no que se refere à longevidade e qualidade de vida (Ferreira, P. et al., 2020; Minayo et al., 2018).

Nesta perspectiva, de acordo com a Comissão Interamericana de Direitos Humanos (1994), a violência contra a mulher (VCM) consiste numa violação dos direitos humanos e liberdades fundamentais, que abole total ou parcialmente a observância, o proveito e a prática de tais direitos e liberdade feminina. Ainda, compõe uma ofensa contra a dignidade humana, notando-se a exteriorização das relações de poder historicamente discrepantes entre homens e mulheres, penetrando em diferentes âmbitos sociais, independente da classe, raça ou nível de escolaridade. Diante disso, o banimento dessa condição é indispensável para que haja o desenvolvimento individual e social, além da plena e igualitária participação em todos os âmbitos da vida.

Nesse contexto, segundo Taquette (2015) as mulheres adolescentes/jovens são mais vulneráveis do que as adultas, visto que, soma-se a fatores como, a baixa idade, o acesso restrito aos meios de proteção, a dependência econômica e a menor escolaridade. Para Reis e Santos (2011), a desigualdade de poder nas relações entre adolescentes femininas e seus parceiros é mais proeminente. Nesse sentido, a violência enfrentada por este grupo populacional se revela de diversas formas, tais como: sexual, física, psicológica, maus tratos e negligência, exploração pelo trabalho, entre outras (Ribeiro \& Silva, 2015).

Acredita-se hoje que a maior parte das mulheres que sofreram abuso e assédio sejam adolescentes e crianças (Engel, 2020). Nos dados extraídos da pesquisa do referido autor, nota-se que 60,5\% das vítimas eram menores de 14 anos. Desse índice, metade dos casos ocorreram contra mulheres negras, 36,2\% contra mulheres brancas, e se verificou uma taxa de não informação de 12,2\% sobre a raça/cor. Em 3,1\% dos casos, as vítimas tinham alguma deficiência mental; além do mais, 57,8\% dos casos ocorreram na própria residência das vítimas, número bem mais elevado que os dados da vitimização. Ainda, de 
acordo com o estudo, as adolescentes que sofrem estupro, $32,2 \%$ dos casos foram praticados por amigos e conhecidos, $12,3 \%$ por padrastos, $11,8 \%$ pelos próprios pais e $12,6 \%$ por desconhecidos.

Nesse ínterim, a ocorrência da VCM está associada a diversos fatores de riscos, incluindo neles as características sociodemográficas e socioeconômicas, o fato de ter presenciado violência familiar na infância, bem como uso de substâncias. Diante dos fatos apresentados, é notório a complexidade e os variados aspectos para que ocorra a VCM, e essa complexidade pode ser o produto entre a interação: indivíduo, família, comunidade e fatores de nível social. Não obstante, se as características sociodemográficas das mulheres em situação de violência dos agressores não forem avaliadas, as ações propostas para diminuir esse fenômeno social pode não surtir efeito (Bernardino et al., 2016; Raine, 2015).

No Brasil, nos últimos anos, diante da dimensão do problema que é a VCM, foram implementados programas voltados para prestação de assistência ao público feminino, cita-se as delegacias de defesa da mulher, os abrigos e os centros de referência de caráter multiprofissional que têm como ênfase, especialmente, a violência sexual e física. Além disso, na última década, surgiram os serviços de atenção à violência sexual para a prevenção e profilaxia de doenças sexualmente transmissíveis (DST), como também a gravidez indesejada (Curia et al., 2020; Pinto et al., 2017). Nesse contexto, para diminuir ou acabar a VCM, além das medidas punitivas, é importante a implementação de ações preventivas, como também medidas de apoio que proporcione a mulher assistência em saúde, jurídica e social (Souza, T., et al., 2018).

A realização desta pesquisa justifica-se pela escassez de trabalhos científicos nas principais bases de dados, e para obtenção de dados acerca da violência cometida contra as mulheres. A partir do panorama exposto, portanto, o objetivo desse artigo é descrever as características dos casos de violência contra crianças e adolescentes do sexo feminino entre 10 e 19 anos notificados na região Nordeste do Brasil nos anos de 2015 a 2019.

\section{Metodologia}

Trata-se de um estudo ecológico, descritivo, de caráter quantitativo, em que a coleta de dados ocorreu entre março e abril de 2021. Para Souza e Kerbauy (2017), os métodos quantitativos são baseados na medida numérica com a análise de poucas variáveis objetivas, com ênfase na comparação dos resultados e emprego de métodos estatísticos.

Os dados foram colhidos através da busca no Sistema de Informação de Agravos de Notificação (SINAN), fornecidos pelo Departamento de Informática do Sistema Único de Saúde (DATASUS). Destacando as notificações por violência no Nordeste do Brasil, nos anos de 2015 a 2019, com análise de 2015-2016 e 2018-2019. A seleção das variáveis integradas dispôs como bases categóricas voltadas para as características relacionadas à vítima, quanto ao agressor e aspectos da violência.

Para compor a parte teórica do estudo, foram utilizados estudos disponíveis nas principais bases de dados online: a Biblioteca Virtual de Saúde (BVS), Scientific Electronic Library Online (SciELO), National Library of Medicine and National Institute of Health U.S. (PubMed) e na Literatura Latino-americana e do Caribe em Ciências da Saúde (LILACS), nos idiomas inglês, português e espanhol entre os anos de 2013 e 2021. As buscas foram realizadas no mês maio de 2021, utilizando os seguintes descritores: violência, mulher, crianças, adolescentes e notificação; com auxílio dos operadores booleanos "AND" e "OR". Além disso, foi conduzida uma pesquisa geral no mecanismo de busca do Google para localizar quaisquer fontes adicionais da literatura cinza.

Foram analisadas as variáveis relacionadas à vítima: características sociodemográficas (raça/cor, escolaridade) com variável fixa faixa etária de 10-19 anos, pois essa faixa etária é mais vulnerável e exposta socialmente, uma vez que sofrem com as desigualdades socioeconômicas. Em seguida, verificaram-se as associadas ao agressor, que consiste na relação com a vítima (familiar, amigo/conhecido, desconhecido, patrão/chefe, cuidador, pessoas com relacionamento institucional, policial/agente da lei, própria pessoa, outros vínculos, etc.). Por fim, as variáveis relacionadas às características da violência, 
que consistem na natureza da violência (física, sexual, negligência/abandono, psicológica/moral, outra violência) de acordo com a classificação da Organização Mundial da Saúde (2002), meio usado na agressão (ameaça, força corporal/espancamento, arma, etc.) e local da agressão (residência, habitação coletiva, via pública, etc.).

Os dados foram retirados do DATASUS. No primeiro momento foram usadas as variáveis: raça/cor, escolaridade e faixa etária 1, os filtros empregados foram faixa etária 10-14 e 15-19 anos, sexo feminino e região Nordeste. No segundo momento, foram usadas as variáveis relacionadas ao agressor e aspectos da violência, aplicadas por cada categoria pertencente a este grupo.

Para realizar o agrupamento dos dados do SINAN, foi utilizado o software Microsoft Excel ${ }^{\circledR}$ versão 2019. Os dados foram então organizados em planilhas com base na correlação entre as variáveis supracitadas e os anos delimitados. Ainda, os valores foram tabulados no formato da frequência absoluta, percentual e valores totais, a fim de proporcionar uma melhor análise e descrição das informações encontradas.

Ressalta-se que este estudo seguiu as orientações estabelecidas pela Resolução $n^{\circ}$ 510/16 (Brasil, 2016), ao dispor sobre a utilização de dados de domínio público com participantes não identificados.

\section{Resultados}

Entre os anos de 2015 e 2019, foram registradas 34.355 notificações de VCM, com idade de 10 a 19 anos, no Nordeste do Brasil. Ao analisar os anos, observou-se que houve crescimento acentuado e gradativo no número de notificações, em 2019, as notificações de violência ficaram em destaque com 12.474 casos, representando 36,3\% do quantitativo total. Com relação aos estados, Pernambuco se sobressaiu com 30\% ( $\mathrm{n}=10.320)$, seguido de Bahia e Ceará, com 17,5\% ( $\mathrm{n}=6.027)$ e $14,6 \%(n=5.032)$, respectivamente (Tabela 1$)$. 
Tabela 1 - Notificações de violência contra mulheres de 10 a 19 anos no Nordeste do Brasil por estado de residência de 20152019.

\begin{tabular}{|c|c|c|c|c|c|c|c|c|c|c|}
\hline \multirow{3}{*}{ Estado de residência } & \multicolumn{10}{|c|}{ Ano de notificação } \\
\hline & \multicolumn{2}{|c|}{2015} & \multicolumn{2}{|c|}{2016} & \multicolumn{2}{|c|}{2018} & \multicolumn{2}{|c|}{2019} & \multicolumn{2}{|c|}{ Total } \\
\hline & $\mathbf{N}$ & $\%$ & $\overline{\mathbf{N}}$ & $\%$ & $\mathbf{N}$ & $\%$ & $\overline{\mathbf{N}}$ & $\%$ & $\overline{\mathbf{N}}$ & $\%$ \\
\hline Alagoas & 504 & 9,0 & 539 & 9,0 & 867 & 8,7 & 1.309 & 10,5 & 3.219 & 9,3 \\
\hline Bahia & 1.233 & 21,4 & 1.359 & 22,0 & 1.738 & 17,5 & 1.697 & 13,6 & 6.027 & 17,5 \\
\hline Ceará & 615 & 10,7 & 847 & 13,6 & 1.478 & 15,0 & 2.092 & 16,7 & 5.032 & 14,6 \\
\hline Maranhão & 353 & 6,1 & 306 & 5,0 & 608 & 6,1 & 1.006 & 8,0 & 2.273 & 6,6 \\
\hline Paraíba & 366 & 6,3 & 198 & 3,1 & 506 & 5,0 & 772 & 6,1 & 1.842 & 5,3 \\
\hline Pernambuco & 1.712 & 29,7 & 1.875 & 30,2 & 3.161 & 31,8 & 3.572 & 28,6 & 10.320 & 30,0 \\
\hline Piauí & 554 & 9,6 & 657 & 10,6 & 777 & 8,0 & 941 & 7,5 & 2.929 & 8,5 \\
\hline Rio Grande do Norte & 240 & 4,1 & 280 & 4,5 & 525 & 5,2 & 751 & 6,0 & 1.796 & 5,2 \\
\hline Sergipe & 181 & 3,1 & 129 & 2,0 & 273 & 2,7 & 334 & 3,0 & 917 & 3,0 \\
\hline Total & 5.758 & 100 & 6.190 & 100 & 9.933 & 100 & 12.474 & 100 & 34.355 & 100 \\
\hline
\end{tabular}

Fonte: SINAN (2021).

Na Tabela 2 é possível observar a prevalência das notificações das mulheres com idade de 15 a 19 anos, com 58,8\% dos casos $(n=20.185)$, apesar de que o presente estudo tratou de faixa etária específica. As notificações referentes a denominação raça/cor da pele, mulheres da cor parda 65,4\% $(n=22.463)$ e branca 12,6\% $(n=4.313)$. A respeito da escolaridade houve maior número de notificações das mulheres com $5^{\mathrm{a}}$ a $8^{\mathrm{a}}$ série incompleta do Ensino fundamental e Ensino médio incompleto, com 28,0\% $(n=9.565)$ e 14,3\% $(n=4.911)$, respectivamente. 
Tabela 2 - Notificações de violência contra mulheres de 10 a 19 anos no Nordeste do Brasil por raça/cor da pele, escolaridade e faixa etária de 2015-2019.

\begin{tabular}{|c|c|c|c|c|c|c|c|c|c|c|}
\hline \multirow{3}{*}{ Variáveis } & \multicolumn{10}{|c|}{ Ano de notificação } \\
\hline & \multicolumn{2}{|c|}{2015} & \multicolumn{2}{|c|}{2016} & \multicolumn{2}{|c|}{2018} & \multicolumn{2}{|c|}{2019} & \multicolumn{2}{|c|}{ Total } \\
\hline & $\mathbf{N}$ & $\%$ & $\mathbf{N}$ & $\%$ & $\mathbf{N}$ & $\%$ & $\mathbf{N}$ & $\%$ & $\mathbf{N}$ & $\%$ \\
\hline \multicolumn{11}{|l|}{ Raça/cor da pele } \\
\hline Parda & 3.357 & 58,3 & 3.679 & 59,4 & 6.770 & 68,1 & 8.657 & 69,5 & 22.463 & 65,4 \\
\hline Branca & 632 & 11,0 & 641 & 10,4 & 1.273 & 12,8 & 1.767 & 14.0 & 4.313 & 12,6 \\
\hline Preta & 444 & 7,7 & 461 & 7,4 & 743 & 7,5 & 876 & 7,0 & 2.524 & 7,3 \\
\hline Amarela & 39 & 0,7 & 58 & 1,0 & 96 & 1,0 & 135 & 1,0 & 328 & 1,0 \\
\hline Indígena & 26 & 0,5 & 22 & 0,4 & 56 & 0,6 & 103 & 1.0 & 207 & 0,6 \\
\hline Ignorado/em branco & 1.260 & 21,8 & 1.329 & 21,4 & 995 & 10,0 & 936 & 7,5 & 4.520 & 13,1 \\
\hline Total & 5.758 & 100 & 6.190 & 100 & 9.933 & 100 & 12.474 & 100 & 34.355 & 100 \\
\hline
\end{tabular}

\section{Escolaridade}

\begin{tabular}{|c|c|c|c|c|c|c|c|c|c|c|}
\hline Analfabeto & 25 & 0,4 & 22 & 0,4 & 29 & 0,3 & 35 & 0,3 & 111 & 0,3 \\
\hline $\begin{array}{l}1^{\mathrm{a}} \text { a } 4^{\mathrm{a}} \text { série incompleta } \\
\text { do Ensino fundamental }\end{array}$ & 288 & 5,0 & 300 & 4,8 & 381 & 3,8 & 410 & 3,3 & 1.379 & 4,0 \\
\hline $\begin{array}{l}4^{\mathrm{a}} \text { série completa do } \\
\text { Ensino fundamental }\end{array}$ & 172 & 3,0 & 171 & 2,8 & 331 & 3,3 & 342 & 2,7 & 1.016 & 3,0 \\
\hline $\begin{array}{l}5^{\mathrm{a}} \text { a } 8^{\mathrm{a}} \text { série incompleta } \\
\text { do Ensino fundamental }\end{array}$ & 1.625 & 28,2 & 1.630 & 26,3 & 2.828 & 28,5 & 3.482 & 28,0 & 9.565 & 28,0 \\
\hline $\begin{array}{l}\text { Ensino fundamental } \\
\text { completo }\end{array}$ & 240 & 4,1 & 287 & 4,6 & 596 & 6,0 & 724 & 5,8 & 1.847 & 5,4 \\
\hline $\begin{array}{l}\text { Ensino médio } \\
\text { incompleto }\end{array}$ & 645 & 11,2 & 644 & 10,4 & 1.507 & 15,2 & 2.115 & 17,0 & 4.911 & 14,3 \\
\hline $\begin{array}{l}\text { Ensino médio } \\
\text { completo }\end{array}$ & 243 & 4,0 & 242 & 3,9 & 519 & 5,2 & 800 & 6,4 & 1.804 & 5,2 \\
\hline $\begin{array}{l}\text { Educação superior } \\
\text { incompleta }\end{array}$ & 59 & 1,0 & 51 & 0,8 & 113 & 1,1 & 164 & 1,3 & 387 & 1,1 \\
\hline $\begin{array}{l}\text { Educação superior } \\
\text { completa }\end{array}$ & 9 & 0,1 & 11 & 0,2 & 11 & 0,1 & 25 & 0,2 & 56 & 0,1 \\
\hline $\begin{array}{l}\text { Ignorado/branco/não } \\
\text { se aplica }\end{array}$ & 2.452 & 43,0 & 2.832 & 45,8 & 3.618 & 36,5 & 4.377 & 35,0 & 13.279 & 38,6 \\
\hline Total & 5.758 & 100 & 6.190 & 100 & 9.933 & 100 & 12.474 & 100 & 34.355 & 100 \\
\hline
\end{tabular}

\section{Faixa etária}

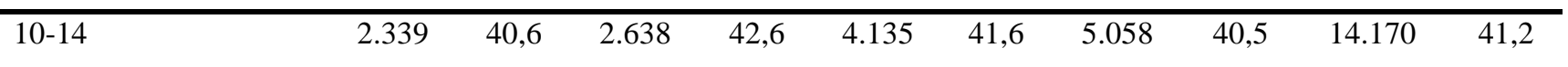




\begin{tabular}{lcccccccccc}
\hline $15-19$ & 3.419 & 59,4 & 3.552 & 57,4 & 5.798 & 58,4 & 7.416 & 59,5 & 20.185 & 58,8 \\
\hline Total & $\mathbf{5 . 7 5 8}$ & $\mathbf{1 0 0}$ & $\mathbf{6 . 1 9 0}$ & $\mathbf{1 0 0}$ & $\mathbf{9 . 9 3 3}$ & $\mathbf{1 0 0}$ & $\mathbf{1 2 . 4 7 4}$ & $\mathbf{1 0 0}$ & $\mathbf{3 4 . 3 5 5}$ & $\mathbf{1 0 0}$ \\
\hline
\end{tabular}

Fonte: SINAN (2021).

A maioria das agressões era autoprovocada 27,7\% (n=9.494) seguido de agressão causada por amigo/conhecido $13,1 \%(n=4.526)$. As informações sobre os prováveis agressores também apresentaram alto percentual de indivíduos que apresentam proximidade com a vítima (Tabela 3). Destaca-se que 8,6\% ( $\mathrm{n}=2.503)$ das notificações de violência foram cometidas pelo pai/padrasto, e 7,2\% ( $\mathrm{n}=2.967)$ por cônjuge.

Tabela 3 - Notificações de violência contra mulheres de 10 a 19 anos no Nordeste do Brasil por vínculo com o agressor de 2015-2019.

\begin{tabular}{|c|c|c|c|c|c|c|c|c|c|c|}
\hline \multirow{3}{*}{$\begin{array}{c}\text { Vínculo com o } \\
\text { agressor }\end{array}$} & \multicolumn{10}{|c|}{ Ano de notificação } \\
\hline & \multicolumn{2}{|c|}{2016} & \multicolumn{2}{|c|}{2017} & \multicolumn{2}{|c|}{2018} & \multicolumn{2}{|c|}{2019} & \multicolumn{2}{|c|}{ Total } \\
\hline & $\overline{\mathbf{N}}$ & $\%$ & $\overline{\mathbf{N}}$ & $\%$ & $\overline{\mathbf{N}}$ & $\%$ & $\overline{\mathbf{N}}$ & $\%$ & $\overline{\mathbf{N}}$ & $\%$ \\
\hline Pai/padrasto & 578 & 10,0 & 447 & 7,2 & 926 & 9,4 & 1.016 & 8,1 & 2.967 & 8,6 \\
\hline Mãe/madrasta & 350 & 6,0 & 467 & 7,5 & 592 & 6,0 & 565 & 4,5 & 1.974 & 5,7 \\
\hline Irmão(a) & 128 & 2,2 & 122 & 2,0 & 211 & 2,1 & 214 & 2,0 & 675 & 2,0 \\
\hline Filho(a) & 11 & 0,2 & 2 & - & 4 & - & 14 & 0,1 & 31 & - \\
\hline Namorado(a) & 483 & 8,4 & 540 & 8,9 & 891 & 9,0 & 951 & 7,6 & 2.865 & 8,3 \\
\hline Ex-namorado(a) & 133 & 2,3 & 137 & 2,2 & 185 & 2,0 & 202 & 1,6 & 657 & 2,0 \\
\hline Cônjuge & 388 & 6,7 & 432 & 7,0 & 875 & 9.0 & 808 & 6,4 & 2.503 & 7,2 \\
\hline Ex-cônjuge & 147 & 2,5 & 157 & 2,5 & 198 & 2,0 & 171 & 1,3 & 673 & 2,0 \\
\hline Amigo/conhecido & 1.007 & 17,5 & 920 & 15,0 & 1.235 & 12,4 & 1.364 & 11,0 & 4.526 & 13,1 \\
\hline Desconhecido(a) & 806 & 14,0 & 821 & 13,2 & 1.005 & 10,1 & 977 & 8,0 & 3.609 & 10,5 \\
\hline Patrão/chefe & 13 & 0,2 & 9 & 0,1 & 7 & - & 8 & - & 37 & 0,1 \\
\hline Cuidador(a) & 14 & 0,2 & 4 & - & 14 & 0,1 & 14 & 0,1 & 46 & 0,1 \\
\hline $\begin{array}{l}\text { Pessoa com } \\
\text { relacionamento } \\
\text { institucional }\end{array}$ & 21 & 0,3 & 26 & 0,4 & 43 & 0,4 & 37 & 0,3 & 127 & 0,3 \\
\hline Policial/agente da Lei & 19 & 0,3 & 18 & 0,3 & 42 & 0,4 & 30 & 0,2 & 109 & 0,3 \\
\hline Própria pessoa & 680 & 12,0 & 853 & 13,8 & 2.829 & 28,4 & 5.132 & 41,1 & 9.494 & 27,7 \\
\hline Outros vínculos & 297 & 5,2 & 335 & 5,4 & 335 & 3,3 & 648 & 5,2 & 1.615 & 5,0 \\
\hline Ignorado/em branco & 683 & 12,0 & 900 & 14,5 & 541 & 5,4 & 323 & 2,5 & 2.447 & 7,1 \\
\hline Total & 5.758 & 100 & 6.190 & 100 & 9.933 & 100 & 12.474 & 100 & 34.355 & 100 \\
\hline
\end{tabular}


Fonte: SINAN (2021).

Em relação à natureza da agressão, houve maior frequência a violência sexual 32,2\% (n=11.083), seguida da violência e física $28,5 \%(n=9.823)$ e outros tipos de violência $22,3 \%(n=7.666)$. O meio usado na agressão teve maior prevalência força corporal/espancamento, com 30,7\% ( $\mathrm{n}=10.545)$ ocorrências, porém com alto percentual de informação ignorada destas variáveis $18 \%(n=6.147)$ casos notificados. Quanto ao local de agressão, a maioria da violência ocorreu na residência da vítima, com 59\% ( $\mathrm{n}=20.183$ ) notificações (Tabela 4).

Tabela 4 - Notificações de violência contra mulheres de 10 a 19 anos no Nordeste do Brasil por natureza da violência, meio usado na agressão e Local da agressão de 2015-2019.

\begin{tabular}{|c|c|c|c|c|c|c|c|c|c|c|}
\hline \multirow{3}{*}{ Variáveis } & \multicolumn{10}{|c|}{ Ano de notificação } \\
\hline & \multicolumn{2}{|c|}{2015} & \multicolumn{2}{|c|}{2016} & \multicolumn{2}{|c|}{2018} & \multicolumn{2}{|c|}{2019} & \multicolumn{2}{|c|}{ Total } \\
\hline & $\mathbf{N}$ & $\%$ & $\mathbf{N}$ & $\%$ & $\mathbf{N}$ & $\%$ & $\mathbf{N}$ & $\%$ & $\mathbf{N}$ & $\%$ \\
\hline \multicolumn{11}{|c|}{ Natureza da violência } \\
\hline Física & 1.987 & 34,5 & 2.052 & 33,1 & 2.395 & 24,1 & 3.389 & 27,1 & 9.823 & 28,5 \\
\hline Sexual & 1.981 & 34,4 & 2.198 & 35,5 & 3.322 & 33,4 & 3.582 & 29,0 & 11.083 & 32,2 \\
\hline Negligencia/ & 281 & 5,0 & 471 & 7,6 & 461 & 4,6 & 383 & 3,0 & 1.596 & 4,6 \\
\hline abandono & & & & & & & & & & \\
\hline Psicológica/moral & 635 & 11,0 & 612 & 9,8 & 863 & 8,8 & 891 & 7,1 & 3.001 & 8,6 \\
\hline Outra violência & 467 & 8,1 & 440 & 7,1 & 2.616 & 26,3 & 3.943 & 31,6 & 7.666 & 22,3 \\
\hline $\begin{array}{l}\text { Ignorado/em } \\
\text { branco }\end{array}$ & 407 & 7,0 & 417 & 7,0 & 276 & 2,8 & 286 & 2,3 & 1.386 & 3,8 \\
\hline Total & 5.758 & 100 & 6.190 & 100 & 9.933 & 100 & 12.474 & 100 & 34.355 & 100 \\
\hline \multicolumn{11}{|c|}{ Meio usado na agressão } \\
\hline Ameaça & 956 & 16,6 & 902 & 14,5 & 1.309 & 13,1 & 1.475 & 12,0 & 4.642 & 13,5 \\
\hline $\begin{array}{l}\text { Força } \\
\text { corporal/espanca } \\
\text { mento }\end{array}$ & 2.279 & 39,5 & 2.347 & 38,0 & 2.911 & 29,3 & 3.008 & 24,2 & 10.545 & 30,7 \\
\hline $\begin{array}{l}\text { Objeto } \\
\text { perfurocortante }\end{array}$ & 537 & 9,4 & 515 & 8,3 & 1.124 & 11,3 & 1.888 & 15,1 & 4.064 & 11,8 \\
\hline $\begin{array}{l}\text { Objeto } \\
\text { contundente }\end{array}$ & 198 & 3,4 & 218 & 3,5 & 276 & 2,8 & 361 & 2,8 & 1.053 & 3,0 \\
\hline Enforcamento & 131 & 2,2 & 156 & 2,5 & 294 & 2,9 & 368 & 3,0 & 949 & 2,7 \\
\hline $\begin{array}{l}\text { Substância ou } \\
\text { objeto quente }\end{array}$ & 42 & 0,8 & 41 & 0,6 & 71 & 1,0 & 131 & 1,0 & 285 & 1,0 \\
\hline Arma de fogo & 432 & 7,5 & 435 & 7,0 & 384 & 3,8 & 307 & 2,4 & 1.558 & 4,5 \\
\hline Outra & 709 & 12,3 & 786 & 12,6 & 1.622 & 16,3 & 1.995 & 16,0 & 5.112 & 14,8 \\
\hline
\end{tabular}




\begin{tabular}{|c|c|c|c|c|c|c|c|c|c|c|}
\hline $\begin{array}{l}\text { Ignorado/em } \\
\text { branco }\end{array}$ & 474 & 8,2 & 790 & 13,0 & 1.942 & 19,5 & 2.941 & 23,5 & 6.147 & 18,0 \\
\hline Total & 5.758 & 100 & 6.190 & 100 & 9.933 & 100 & 12.474 & 100 & 34.355 & 100 \\
\hline \multicolumn{11}{|c|}{ Local da agressão } \\
\hline Residência & 2.745 & 47,7 & 3.053 & 49,3 & 6.113 & 61,5 & 8.272 & 66,1 & 20.183 & $\overline{59,0}$ \\
\hline $\begin{array}{l}\text { Habitação } \\
\text { coletiva }\end{array}$ & 36 & 0,6 & 38 & 0,6 & 74 & 0,7 & 72 & 0,5 & 220 & 0,6 \\
\hline Escola & 108 & 2,0 & 85 & 1,3 & 205 & 2,0 & 347 & 2,7 & 745 & 2,1 \\
\hline $\begin{array}{l}\text { Local de prática } \\
\text { esportiva }\end{array}$ & 20 & 0,3 & 21 & 0,3 & 27 & 0,3 & 24 & 0,2 & 92 & 0,2 \\
\hline Bar ou similar & 88 & 1,5 & 76 & 1,2 & 125 & 1,2 & 133 & 1,0 & 422 & 1,2 \\
\hline Via pública & 985 & 17,1 & 1.032 & 16,6 & 1.238 & 12,4 & 1.085 & 9,0 & 4.340 & 12,6 \\
\hline $\begin{array}{l}\text { Comércio/ } \\
\text { serviços }\end{array}$ & 53 & 1,0 & 44 & 1,0 & 77 & 1,0 & 107 & 0,8 & 281 & 0,8 \\
\hline $\begin{array}{l}\text { Indústrias/ } \\
\text { construção }\end{array}$ & 14 & 0,2 & 7 & 0,1 & 7 & - & 12 & - & 40 & 0,1 \\
\hline Outros & 428 & 7,4 & 502 & 8,1 & 690 & 6,9 & 713 & 6,0 & 2.333 & 6,8 \\
\hline $\begin{array}{l}\text { Ignorado/ } \\
\text { em branco }\end{array}$ & 1.281 & 22,2 & 1.332 & 21,5 & 1.377 & 14,0 & 1.709 & 13,7 & 5.699 & 16,6 \\
\hline Total & 5.758 & 100 & 6.190 & 100 & 9.933 & 100 & 12.474 & 100 & 34.355 & 100 \\
\hline
\end{tabular}

Fonte: SINAN (2021).

\section{Discussão}

De modo geral, a VCM é um tópico que tem grande relevância social, visto que estas mulheres encontram-se em estado de exposição constante necessitando de atenção dos governantes e da população em geral (Campos et al., 2020). No entanto, observa-se o constante crescimento do número de notificações. Com isso surgem inúmeras iniciativas dos serviços especializados de atendimento à mulher que estimulam a assistência integral a esse público, e vem demonstrando um crescente interesse em diminuir a prática de crimes contra a população feminina, tornando a VCM uma problemática de saúde pública (Scott et al., 2016; Ferreira Junior et al., 2021).

Em virtude dos dados apresentados neste estudo, foi possível observar que a violência com uso da força corporal e espancamento demonstraram-se elevados, representando um terço dos casos notificados. Os dados são convergentes com a pesquisa de Mascarenhas et al. (2020) realizada entre 2010 e 2017, em território nacional, onde 86,6\% das vítimas sofreram violência física. No que diz respeito à violência sexual, esta apresentou-se em evidência, tendo $32,2 \%$ do total das notificações, estando em concordância com o estudo de Gaspar e Pereira (2018) que retratou a violência sexual como a mais frequente com $67,2 \%$.

Embora a variável escolaridade não tenha sido registrada com integridade no sistema, visto que a faixa etária inserida na pesquisa não permite elevados níveis das categorias, mesmo assim, a categoria que deteve de maior frequência foi a de nível 
de escolaridade apontando que as mulheres haviam cursado da $5^{\text {a }}$ a $8^{a}$ série incompleta. Além disso, Silva e Oliveira, (2016) afirmaram em sua pesquisa, que as vítimas apresentaram diferentes níveis de instrução, apesar de que o baixo nível de escolaridade, frequentemente, surge como um fator de risco para as mulheres expostas à violência.

A presente pesquisa evidenciou uma elevada incidência de VCM em ambiente domiciliar e via pública, como apontou a investigação de Teofilo et al. (2019) realizada em Niterói/Rio de Janeiro (48,5\% dos episódios de VCM nas residências e via pública, com 20,5\%.). Dessa forma, os dados encontrados demostram que a mulher pode correr risco tanto em domicílio quanto em via pública, expondo-se a sofrer violência em ambientes tidos como seguros.

Destaca-se ainda que, houve notificações significativas com meio de agressão sob o uso de objetos perfurocortantes e contundente predominou, esses dados tem importante relevância, uma vez que quando comparado aos demais meios, apresenta alta possibilidade de ter como desfecho, o óbito (Andrade et al., 2016; Ferreira, R., et al., 2016; Leite et al., 2017). Nesse cenário, as categorias destacadas acima apresentaram-se como meio comum de agressão encontrada nesta pesquisa, como também apontado por Silva e Oliveira (2016) em uma pesquisa realizada na cidade de Brasília/Distrito Federal, estimando-se entre $3,2 \%$ de chances de óbito.

No que se refere à faixa etária das mulheres vítimas de violência, observou-se que diante das notificações analisadas, a idade de 15 a 19 anos caracteriza-se pela maior prevalência de casos (58,8\%), haja vista o público adolescente delimitado para este estudo. Corroborando com tais achados, o estudo de Souto et al. (2018) evidenciou que as jovens dentro da faixa etária supracitada são as principais vítimas de violência, em uma população amostral que engloba desde menores de 1 ano a 19 anos na cidade de Minas Gerais, entre os anos de 2013 e 2015. Assim, nota-se a ocorrência de vulnerabilidade infantojuvenil em ambiente domiciliar praticado por indivíduos próximos.

Posteriormente, contemplando a correlação entre os estados do Nordeste e os anos determinados para o desenvolvimento deste estudo (2015-2019), torna-se perceptível que Pernambuco, sucedido da Bahia e Ceará, apresentaram respectivamente 30\% ( $\mathrm{n}=10.320), 17,5 \%(\mathrm{n}=6.027)$ e 14,6\% ( $\mathrm{n}=5.032)$. Ainda, nesse cenário destaca-se Sergipe com 3\% ( $\mathrm{n}$ = 917) caracterizando o menor quantitativo das taxas de notificações de VCM na faixa etária de 10 a 19 anos de idade. Em conformidade com essas informações, Araújo et al. (2019) reafirmou a elevada incidência de violência nos estados de Pernambuco e Bahia, respectivamente, ressaltando a discrepância entre os seus quantitativos de casos para com os demais estados. Nesse contexto, ressalta-se acerca da ampla extensão populacional que os mesmos detêm, além da cultura misógina enraizada, que promove a concepção errônea de que o homem se denomina por uma figura superior à mulher.

Em ressalva ao público-alvo e continuamente aos aspectos culturais integrados na sociedade, faz-se relevante discutir sobre o contexto histórico (Garcia et al., 2017). Esses autores afirmam que na época colonial no Brasil e no mundo oferecer um ambiente afetivo e seguro para crianças e adolescentes não era relevante ou prioridade da família. Sendo assim, de acordo com Leite et al. (2017), tais pontuações contribuíram para a intensificação do abuso, exploração e violência infanto-juvenil.

Em contrapartida, incialmente com a promulgação da Constituição Federal de 1988 (Brasil, 1988), em seguida pela implementação da Lei Federal no 8.069/90 do Estatuto da Criança e do Adolescente (Brasil, 1990) e do Plano Nacional de Enfrentamento da Violência Sexual Infantojuvenil (Brasil, 2013), foi realizado um maior combate às adversidades perpassadas pelas crianças e adolescentes. Assim, houve uma melhor atribuição de direitos e da garantia de deveres acrescidos às responsabilidades do Estado, da sociedade e da família (Garcia et al., 2017). Além disso, destaca-se a garantia do registro e acompanhamento das notificações nos sistemas de informações, com a finalidade de verificar o cumprimento ou violação dos direitos estabelecidos. Dessa forma, permitindo a intervenção necessária dos órgãos responsáveis (Leite et al., 2017).

Com base no que já foi discutido, cabe destacar a relevância dos profissionais no atendimento a essas mulheres e a sua notificação (Garcia et al., 2017). No Brasil, o Sistema de Informações sobre Mortalidade (SIM) revela que o número de óbitos por violência apresenta taxas de crescimento significativas, todavia, apontam-se limitações nesse sistema, sendo que o mais 
utilizado atualmente para os registros desses casos é o SINAN, através da Ficha de Notificação para Violência Doméstica, Sexual e/ou outras Violências (Kind et al., 2013).

Ressalta-se que a Lei $\mathrm{n}^{\circ}$ 10.778, regulamenta a notificação compulsória, em todo o território nacional, de casos suspeitos ou confirmados de VCM atendidos em serviços de saúde públicos ou privados (Brasil, 2003). No entanto, observouse que um dos motivos associados à subnotificação é justamente a dificuldade de identificação do agravo por parte dos profissionais de saúde. Sendo assim, a pesquisa conduzida por Gomes e Erdmann (2014) apontou que os profissionais que atuam na estratégia de saúde da família (ESF) em Florianópolis/Santa Catarina, apesar de demonstrarem conhecimento teórico referente à situação de VCM, apresentaram dificuldades em identificá-la como motivo para a busca de atendimento na unidade.

\section{Conclusão}

Neste artigo, foi possível evidenciar os impactos das diferentes formas de VCM com idade de 10 a 19 anos no Nordeste. Percebe-se pelo alto quantitativo observado de notificações, que as características apresentadas reforçam que as mulheres se encontram com baixo nível educacional. Dado que, na maioria das vezes, aspectos sociais como analfabetismo, baixa educação, somados a pouca oportunidade profissional ou desemprego, parecem exacerbar a magnitude do problema.

Nesse contexto, ressalta-se a importância da aplicabilidade de metodologias de segurança e prevenção da violência, além da construção de políticas públicas efetivas de combate à VCM contra crianças e adolescentes do sexo feminino, visto que elas se encontram em estado de maior suscetibilidade. Uma vez que, estão expostas tanto no contexto domiciliar quanto em espaço externo, destacando-se a via pública. Além disso, pressupõe-se que as subnotificações podem ser influenciadas pelo causador da agressão, dado que a maior prevalência de violência cometida foi por indivíduos próximos das vítimas.

Sendo assim, a VCM é um fator de ameaça à vida que não está somente ligado à saúde da mulher, mas em todos os eixos da sociedade. Então é essencial que os profissionais de saúde sejam capacitados para identificar mulheres em situação de violência, para que possam realizar deliberações e prestar a assistência necessária, integrando também as redes de atenção e apoio social.

Finalmente, expõe-se a importância desse artigo como estratégia de levantar novos dados referentes a violência contra mulheres com idade entre 10 e 19 anos registrados rotineiramente nos estados do Nordeste. Ainda, propõe-se o crescimento do alcance de pesquisas futuras relacionadas a essa temática com o objetivo de somar aos estudos existentes para o fortalecimento da literatura nacional, assim como identificar as características das agressões e os ambientes no qual é cometido, dispondo-se como referência para criação e execução de políticas públicas.

\section{Referências}

Andrade, J. O., Castro, S. S., Heitor, S. F. D., Andrade, W. P., \& Atihe, C. C. (2016). Indicadores da violência contra a mulher provenientes das notificações dos serviços de saúde de minas gerais-Brasil. Texto e Contexto Enfermagem, 25(3), 1-9. https://doi.org/10.1590/0104-07072016002880015

Araújo, B. F., Pereira, F. M. F., Freitas, P. V. L., Saturnino, V. S., \& Santos, E. V. L. (2019). Análise da prevalência dos tipos de violência contra a mulher na região nordeste. Journal of Medicine and Health Promotion, 4(1), 1086-1095. http://jmhp.fiponline.edu.br/pdf/cliente=13be613bb5bc1c39e88896699aa4c636b3.pdf

Bernardino, Í. M., Barbosa, K. G. N., Nóbrega, L. M., Cavalcante, G. M. S., Ferreira, E. F. e, \& d'Avila, S. (2016). Violência contra mulheres em diferentes estágios do ciclo de vida no Brasil: Um estudo exploratório. Revista Brasileira de Epidemiologia, 19(4), 740-752. https://doi.org/10.1590/19805497201600040005

Brasil. (1988). Constituição da República Federativa do Brasil de 1988. Brasília: Distrito Federal. planalto.gov.br/ccivil_03/constituicao/constituicao.htm

Brasil. (2003). Lei $n^{o} 10.778$, de 24 de novembro de 2003. Estabelece a notificação compulsória, no território nacional, do caso de violência contra a mulher que for atendida em serviços de saúde públicos ou privados. Brasília: Distrito Federal. http://www.planalto.gov.br/ccivil_03/leis/2003/110.778.htm

Brasil. (1990). Lei $n^{\circ}$ 8.069, de 13 de julho de 1990. Dispõe sobre o Estatuto da Criança e do Adolescente e dá outras providências. Brasília: Distrito Federal. http://www.planalto.gov.br/ccivil_03/leis/18069.htm 
Brasil. (2013). Plano Nacional de Enfrentamento da Violência Sexual contra Crianças e Adolescentes. https://www.gov.br/mdh/pt-br/centrais-deconteudo/crianca-e-adolescente/plano-nacional-de-enfrentamento-da-violencia-sexual-contra-criancas-e-adolescentes.pdf/view

Brasil. (2016). Resolução $n^{o} 510$, de 7 de abril de 2016. Conselho Nacional de Saúde. Brasília: Distrito Federal. https://bvsms.saude.gov.br/bvs/saudelegis/cns/2016/res0510_07_04_2016.html

Campos, B., Tchalekian, B., \& Paiva, V. (2020). Violência contra a mulher: vulnerabilidade programática em tempos de Sars-Cov-2/covid-19 em São Paulo. Psicologia \& Sociedade, 32, e020015. https://doi.org/10.1590/1807-0310/2020V32240336

Curia, B. G., Gonçalves, V. D., Zamora, J. C., Ruoso, A., Ligório, I. S., \& Habigzang, L. (2020). Produções Científicas Brasileiras em Psicologia sobre Violência contra Mulher por Parceiro Íntimo. Psicologia: Ciência e Profissão, 40, 1-19. https://doi.org/10.1590/1982-3703003189184

Comissão Interamericana de Direitos Humanos (1994). Convenção interamericana para prevenir, punir e erradicar a violência contra a mulher, "convenção de Belém do Pará". http://www.cidh.org/basicos/portugues/m.belem.do.para.htm

Engel, C. L. (2020). A Violência contra a mulher. Instituto de pesquisa econômica e aplicada. Brasília: ipea. https://www.ipea.gov.br/retrato/pdf/190215_tema_d_a_violenca_contra_mulher.pdf

Ferreira Junior, S. S., Almeida, V. T. S., Júnior, A. L. D., \& Luiz, R. S. (2021). As prisioneiras da dor: argumentando sobre a subnotificação da violência doméstica em meio à pandemia . Brazilian Journal of Development, 7(4), 38721-38739. https://doi.org/10.34117/bjdv7n4-367

Ferreira, P. C., Batista, V. C., Pesce, G. B., Lino, I. G. T., Marquete, V. F., \& Marcon, S. S. (2020). Caracterização dos casos de violência contra mulheres. Revista de Enfermagem UFPE on Line, 14(0), 243993. https://doi.org/10.5205/1981-8963.2020.243583

Ferreira, R. M., Vasconcelos, T. B., Moreira Filho, R. E., \& Macena, R. H. M. (2016). Características de saúde de mulheres em situação de violência doméstica abrigadas em uma unidade de proteção estadual. Ciência \& Saúde Coletiva, 21(12), 3937-3946. https://doi.org/10.1590/1413812320152112.09092015

Garcia, P. A., Macieira, A. P., \& Oliveira, D. E. S. D. (2017). O trabalho da equipe multiprofissional com crianças e adolescentes vítimas de violência sexual atendidas na unidade hospitalar. Revista LEVS, 20(20), 29-56. https://doi.org/10.36311/1983-2192.2018.v20n20.03.p29

Gaspar, R. S., \& Pereira, M. U. L. (2018). Evolução da notificação de violência sexual no Brasil de 2009 a 2013. Cadernos de Saúde Pública, 34(11), 1-10. https://doi.org/10.1590/0102-311x00172617

Gomes, N. P., \& Erdmann, A. L. (2014). Violência conjugal na perspectiva de profissionais da "Estratégia Saúde da Família": Problema de saúde pública e a necessidade do cuidado à mulher. Revista Latino-Americana de Enfermagem, 22(1), 76-84. https://doi.org/10.1590/0104-1169.3062.2397

Kind, L., Orsini, M. L. P., Nepomuceno, V., Gonçalves, L., Souza, G. A. de., \& Ferreira, M. F. F. (2013). Subnotificação e (in)visibilidade da violência contra mulheres na atenção primária à saúde. Cadernos de Saúde Pública, 29(9), 1805-1815. https://doi.org/10.1590/0102-311X00096312

Leite, F. M. C., Mascarello, K. C., Almeida, A. P. S. C., Fávero, J. L., Santos, A. S., Silva, I. C. M., \& Wehrmeister, F. C. (2017). Análise da tendência da mortalidade feminina por agressão no Brasil, estados e regiões. Ciência \& Saúde Coletiva, 22(9), 2971-2978. https://doi.org/10.1590/141381232017229.25702016

Mascarenhas, M. D. M., Tomaz, G. R., Meneses, G. M. S., Rodrigues, M. T. P., Pereira, V. O. M., \& Corassa, R. B. (2020). Análise das notificações de violência por parceiro íntimo contra mulheres, Brasil, 2011-2017. Revista Brasileira de Epidemiologia, 23, 1-13. https://doi.org/10.1590/1980549720200007.supl.1

Minayo, M. C. S. (2007). Conceitos, teorias e tipologias de violência: a violência faz mal à saúde. Impactos da Violência na Saúde [online]: Editora FIOCRUZ, 21-42.

Minayo, M. C. S., et al. (2018). Institucionalização do tema da violência no SUS: avanços e desafios. Ciência \& Saúde Coletiva, 23(6), $2007-2016$. https://doi.org/10.1590/1413-81232018236.04962018

Organização Mundial de Saúde - OMS, World Report on Violence and Health. Geneve: WHO, 2002. http://apps.who.int/iris/bitstream/10665/42495/1/9241545615_eng.pd

Pinto, L. S. S., Oliveira, I. M. P., Pinto, E. S. S., Leite, C. B. C., Melo, A. N., \& Deus, M. C. B. R. (2017). Políticas públicas de proteção à mulher: avaliação do atendimento em saúde de vítimas de violência sexual. Ciência \& Saúde Coletiva, 22(5), 1501-1508. https://doi.org/10.1590/1413-81232017225.33272016

Raine, A. (2015). A anatomia da violência: as raízes biológicas da criminalidade. Artmed Editora.

Reis, C. B., \& Santos, N. R. (2011). Relações desiguais de gênero no discurso de adolescentes. Ciência \& Saúde Coletiva, 16(10), 3979-3984. https://doi.org/10.1590/S1413-81232011001100002

Ribeiro, M. I. C., \& Silva, M. G. S. N. (2015). Violência, vulnerabilidade e desigualdade socioespacial: análise das dificuldades enfrentadas pelas mulheres vítimas de violência doméstica na área urbana de Porto Velho-RO. Revista Latino-americana de Geografia e Gênero, 6(2), 92-104. http://dx.doi.org/10.5212/Rlagg.v.6.i2.0006

Scott, P., Nascimento, F. S., Cordeiro, R., \& Nanes, G. (2016). Redes de Enfrentamento da Violência contra Mulheres no Sertão de Pernambuco. Revista Estudos Feministas, 24(3), 851-870. https://doi.org/10.1590/1806-9584-2016v24n3p851

Silva, L. E. L., \& Oliveira, M. L. C. (2016). Características epidemiológicas da violência contra a mulher no Distrito Federal, 2009 a 2012. Epidemiologia e Serviços de Saúde : Revista Do Sistema Único de Saúde Do Brasil, 25(2), 331-342. https://doi.org/10.5123/S1679-49742016000200012

Souto, D. F., Zanin, L., Ambrosano, G. M. B., \& Flório, F. M. (2018). Violência contra crianças e adolescentes: perfil e tendências decorrentes da Lei n ${ }^{\circ}$ 13.010. Revista Brasileira de Enfermagem, 71(Supl. 3), 1237-1246. https://doi.org/10.1590/0034-7167-2017-0048 
Research, Society and Development, v. 10, n. 7, e57610716921, 2021

(CC BY 4.0) | ISSN 2525-3409 | DOI: http://dx.doi.org/10.33448/rsd-v10i7.16921

Souza, K. R., \& Kerbauy, M. T. M. (2017). Abordagem quanti-qualitativa: superação da dicotomia quantitativa-qualitativa na pesquisa em educação. Educação e Filosofia, 31(61), 21-44. https://doi.org/10.14393/REVEDFIL.issn.0102-6801.v31n61a2017-p21a44

Souza, T. M. C., \& Rezende, F. F. (2018). Violência contra mulher: concepções e práticas de profissionais de serviços públicos. Estudos Interdisciplinares em Psicologia, 9(2), 21-38. https://doi.org/10.5433/2236-6407.2016v9n1p21

Taquette, S. R. (2015). Violência contra a mulher adolescente - revisão de estudos epidemiológicos brasileiros publicados entre 2006 e 2011 . Adolescência \& Saúde, 12(1), 66-77. http://adolescenciaesaude.com/detalhe_artigo.asp?id=478

Teofilo, M. A., Kale, L. P., Eppinghaus, A. L. P., Azevedo, O. P., Farias, R. S., Maduro Neto, J. P., Costa, A. J. L., \& Cavalcanti, M. L. T. (2019). Violência contra mulheres em Niterói, Rio de Janeiro: informações do Sistema de Vigilância de Violências e Acidentes (2010-2014). Cadernos Saúde Coletiva, 27(4), 437-447. https://doi.org/10.1590/1414-462X201900040302X 